\title{
Estabelecimento de leguminosas arbóreas em pastos de capim-marandu e tanzânia
}

\author{
Paulo Francisco Dias ${ }^{(1)}$, Sebastião Manhães Souto(2), Bruno Campbell de Azevedo(3), Márcia de Souza Vieira( ${ }^{(3)}$, \\ Aline Alves Colombari( ${ }^{(3)}$, Juliana Dias ${ }^{(3)}$ e Avílio Antônio Franco ${ }^{(2)}$ \\ (1)Empresa de Pesquisa Agropecuária do Estado do Rio de Janeiro, Estação Experimental de Seropédica, BR 465, Km 7, \\ CEP 23890-000 Seropédica, RJ. E-mail: pfranciscodias@hotmail.com ${ }^{(2)}$ Embrapa Agrobiologia, BR 465, Km 7, CEP 23890-000 \\ Seropédica, RJ. E-mail: smsouto@cnpab.embrapa.br, avilio@cnpab.embrapa.br (3)Universidade Federal Rural do Rio de \\ Janeiro, BR 465, Km 7, CEP 23890-000 Seropédica, RJ. E-mail: brunocampbell@bol.com.br, msvzootec@yahoo.com.br, \\ alinecolombari@hotmail.com, julianarural@bol.com.br
}

\begin{abstract}
Resumo - O objetivo deste trabalho foi avaliar o desempenho de mudas sem proteção (de cercas ou estacas) de quatro espécies de leguminosas arbóreas e uma mistura eqüitativa dessas espécies, introduzidas em pastagens de Brachiaria brizantha cv. Marandu e Panicum maximum cv. Tanzânia, na presença de gado. O delineamento experimental foi o inteiramente ao acaso, em esquema fatorial 2x5, duas gramíneas (marandu e tanzânia) e quatro espécies de leguminosas (Mimosa artemisiana, Pseudosamanea guachapele, Enterolobium contortisiliquum, Acacia farnesiana e uma mistura dessas espécies), com três repetições. Avaliaram-se: altura da muda, diâmetro do caule, diâmetro da copa, sobrevivência da muda, freqüência de pastejo e ocorrência de formigas. As diferenças estatísticas entre as médias da variável canônica principal, pelo teste de Scott-Knott, indicaram a formação de três agrupamentos, tendo-se destacado o grupo formado pelos tratamentos $M$. artemisiana e mistura de leguminosas, nos dois pastos, mais E. contortisiliquum e A. farnesiana, nos pastos dos capins marandu e tanzânia, respectivamente. Diferenças entre as médias dos tratamentos relativas a cada variável, calculadas por meio de intervalos de confiança de Bonferroni, mostraram que mudas de $M$. artemisiana apresentaram maior altura e sobrevivência em pasto de capim-marandu. Mudas dessa leguminosa, sem proteção, são indicadas para ser introduzidas, nas pastagens de capim-marandu da região, na presença do gado.
\end{abstract}

Termos para indexação: Brachiaria brizantha, Mimosa artemisiana, Panicum maximum, pastejo, sistema silvipastoril, sobrevivência.

\section{Establishment of leguminous trees in marandu and tanzânia pastures}

Abstract - The objective of this work was to evaluate the performance of nonprotected (by fences or pickets) seedlings of leguminous tree species and an equitable mix of these species, introduced in pastures of Brachiaria brizantha cv. Marandu and Panicum maximum cv. Tanzânia, in the presence of cattle. The experimental design was a completely randomized one, in a 2x5 factorial arrangement, with two grasses (marandu and tanzânia) and four leguminous species (Mimosa artemisiana, Pseudomanea guachapele, Enterolobium contortisiliquum, Acacia farnesiana), and a mix of these species, with three replicates. Evaluations were made for: seedling height, stem diameter, canopy diameter, seedling survival, pasture frequency and ant occurrence. The statistical difference between the means of the main canonical variable, by the test of Scott-Knott, indicated the formation of three groups, standing out the one formed by M. artemisiana and mixture of leguminous in the two pastures, plus E. contortisiliquum and A. farnesiana in pastures of marandu and tanzânia grass, respectively. Differences between the means of treatments for each variable, calculated by means of Bonferroni's intervals of confidence, showed that $M$. artemisiana presented the greatest height and seedling survival in the pasture of marandu grass. This leguminous seedlings, without protection, are indicated to be introduced in pastures of marandu grass in the region, in the presence of cattle.

Index terms: Brachiaria brizantha, Mimosa artemisiana, Panicum maximum, grazing, silvipastural system, survival.

\section{Introdução}

Os sistemas silvipastoris combinam espécies lenhosas e produção animal, em alguma forma de arranjo temporal ou espacial, e se constituem em ferramentas importantes do desenvolvimento sustentável, já que combinam produção com conservação dos recursos naturais (hídricos e solo), além de atender às diferentes necessidades dos produtores rurais, como alimento, madeira, lenha, forragem, plantas medicinais e 
fibras. Também auxiliam na conservação dos solos, recuperação de microbacias, recomposição ordenada de áreas florestais e manutenção da biodiversidade (Nicodemo et al., 2004; Dias Filho \& Ferreira, 2007).

As espécies arbóreas podem melhorar a produção, a qualidade e a persistência das pastagens (Alonzo, 2000; Ibrahim et al., 2001), seqüestrar quantidades substanciais de carbono (Macedo et al., 2008) e aumentar a biodiversidade em pastagens (Dias et al., 2006). Esses benefícios são maiores no caso de leguminosas arbóreas que possuem a capacidade de fixar o nitrogênio do ar (Dias, 2005).

Segundo Andrade et al. (2002), uma das limitações para a implantação de sistemas silvipastoris é a dificuldade para introdução das árvores em pastagens estabelecidas. Constatou-se que a implementação do método de proteção das mudas, com arame farpado e uma estaca, implica o acréscimo de aproximadamente 9\% no custo operacional da exploração bovina de corte, o que significa redução de $27 \%$ no retorno bruto (Montoya \& Baggio, 1991).

Portanto, o estabelecimento de leguminosas arbóreas edafoclimatologicamente mais adaptadas, sem a proteção de mudas e na presença de animais, poderá reduzir o custo da arborização e permitir a introdução de espécies, mesmo em sistemas com baixa rentabilidade (Dias, 2005). Nas pastagens das regiões de baixada e serrana do Estado do Rio de Janeiro, foram selecionadas espécies de leguminosas arbóreas que se estabeleceram com sucesso a partir de mudas sem proteção na presença do gado (Dias et al., 2007a, 2007b).

O sucesso da introdução de uma espécie arbórea na pastagem, sem proteção e na presença dos animais, depende do grau de sua aceitabilidade pelos animais (Ash, 1990; Hindrichsen et al., 2004), da velocidade de crescimento e da competição com a pastagem. A aceitabilidade da forrageira, pelos animais, é influenciada pelo teor de tanino (Nozella, 2001; Andersson et al., 2006; Nozella et al., 2006) e, também, pelo fato de a planta já ser parte ou não de sua dieta na pastagem (Souto et al., 1975; Launchbaugh et al., 1993). Os animais pastejam seletivamente em uma pastagem, escolhendo as forrageiras de melhor qualidade e de mais fácil apreensão, para compor a sua dieta (Baumont et al., 2000).

O objetivo deste trabalho foi avaliar o desempenho de mudas sem proteção (de cercas ou estacas) de quatro espécies de leguminosas arbóreas, isoladamente e em uma mistura eqüitativa dessas quatro espécies, introduzidas em duas pastagens estabelecidas com Brachiaria brizantha cv. Marandu e Panicum maximum cv. Tanzânia, na presença de gado.

\section{Material e Métodos}

O trabalho foi conduzido em duas áreas de pastagens, formadas em dezembro de 2005 e janeiro de 2006, respectivamente, com os capins $B$. brizantha cv. Marandu e $P$. maximum cv. Tanzânia, no setor de Bovino de Leite da Empresa de Pesquisa Agropecuária do Estado do Rio de Janeiro (Pesagro), Estação Experimental de Seropédica, Seropédica, RJ (21 ${ }^{\circ} 45^{\prime} \mathrm{S}$, $43^{\circ} 41^{\prime} \mathrm{W}, 33 \mathrm{~m}$ de altitude).

O solo predominante da área experimental é um Planossolo Háplico distrófico arênico, com a seguinte composição química: $\mathrm{pH} 4,6 ; \mathrm{Ca}, 1,5 \mathrm{cmol}_{\mathrm{c}} \mathrm{dm}^{-3}$; $\mathrm{Mg}, 1,3 \mathrm{cmol}_{\mathrm{c}} \mathrm{dm}^{-3}$; K, $14 \mathrm{mg} \mathrm{kg}^{-1}$; P, $19 \mathrm{mg} \mathrm{kg}^{-1}$. A temperatura média, a média das máximas e a média das mínimas e a precipitação pluvial, durante o período experimental, foram $23,6^{\circ} \mathrm{C}, 29,5^{\circ} \mathrm{C}, 19,3^{\circ} \mathrm{C}$ e $2.276 \mathrm{~mm}$, respectivamente.

Nessas pastagens, foram introduzidas mudas (40-60 cm) sem proteção, das espécies de leguminosas arbóreas Mimosa artemisiana Heringer \& Paula (jurema-branca), Pseudosamanea guachapele (Kunth) Harms (albizia), Enterolobium contortisiliquum (Vell) Morong (orelha-de-negro), Acacia farnesiana (L.) Willd (aromita), mais uma mistura eqüitativa dessas espécies, em relação ao número de mudas. As três primeiras espécies foram selecionadas entre 16 , com base em estudos anteriores, por apresentarem potencial para o estabelecimento em pastagens na presença de animais (Dias, 2005).

As mudas foram produzidas no viveiro do campo experimental da Embrapa Agrobiologia, Seropédica, RJ, por meio de sementes com inoculação de estirpes eficientes de rizóbio, recomendadas por Faria (2001), e, também, de uma mistura dos fungos micorrízicos, Gigaspora margarita e Glomus clarum. A inoculação foi feita segundo De-Polli \& Franco (1985). Logo após a inoculação dos fungos, as sementes foram semeadas em sacos de plástico de 800 a $1.000 \mathrm{~g}$, com substrato com $30 \%$ de composto orgânico, $30 \%$ de argila, $30 \%$ de areia e $10 \%$ de fosfato de rocha.

As mudas foram plantadas, após o rebaixamento dos pastos pelos animais, no espaçamento de 10x10 m 
(100 mudas ha-1 ${ }^{-1}$, em 30 piquetes de 50x50 m. Cada espécie e a mistura das quatro espécies foram plantadas em 10/4/2005, em três piquetes com cada gramínea manejados com um lote com 40 novilhas (peso inicial entre 250 e $300 \mathrm{~kg}$ de peso vivo), segundo o método de lotação rotativa, com um dia de ocupação com todas as novilhas por piquete, e 30 dias de descanso, durante todo período experimental. No período de descanso, os animais foram deslocados para as pastagens de capim-marandu ou tanzânia, sem árvores. Durante o período experimental (17/6/2005 a 13/4/2007), tomouse o cuidado para que a oferta de forragem e a pressão de pastejo fossem adequadas, no sentido de que os animais, se fosse o caso, não deixassem de pastejar as mudas das leguminosas arbóreas.

As variáveis estudadas nas mudas das espécies arbóreas foram as seguintes: altura da muda, diâmetro do caule, diâmetro da copa, plantas sobreviventes, freqüência de pastejo e ocorrência de formigas. Nas medições de altura (da superfície do solo ao ápice da planta), de diâmetro do caule (a $10 \mathrm{~cm}$ da superfície do solo) e da copa (projeção da copa no sentido NorteSul e Leste-Oeste), usou-se fita métrica com graduação mínima de $1 \mathrm{~mm}$, em todas as mudas do piquete, por ocasião das avaliações, assim como a contagem de mudas mortas, atacadas por formigas e pastejadas.

$\mathrm{O}$ delineamento experimental utilizado foi $\mathrm{o}$ inteiramente ao acaso, em um esquema fatorial $2 \times 5$, com duas gramíneas (marandu e tanzânia) e cinco tratamentos com leguminosas ( $M$. artemisiana, P. guachapele, E. contortisiliquum, A. farnesiana e uma mistura das quatro espécies), com três repetições em cada gramínea. A correlação de Pearson entre as variáveis foi avaliada pelo programa SAEG (Fundação Arthur Bernardes, 2005). As diferenças dos vetores das médias de tratamentos foram verificadas por variância multivariada Manova, pelos testes de HotellingLawley, Pillai, Wilks e Roy (Ribeiro Junior, 2001), para se testar a hipótese de nulidade, que é a igualdade entre os vetores de médias dos tratamentos.

Com base nos resultados das correlações de Pearson significativas entre as variáveis, na rejeição da hipótese de nulidade pelos quatro testes de MANOVA, e na ausência de normalidade na distribuição das variáveis, a análise dos dados do experimento foi realizada por meio da análise de variância multivariada (PimentelGomes, 2000; Ribeiro Junior, 2001). O agrupamento das variáveis em relação aos tratamentos foi feito por variáveis canônicas, que possuem as mesmas finalidades apresentadas pelos componentes principais e que, entretanto, apresentam a vantagem adicional de se poderem considerar as covariâncias residuais existentes entre as médias dos tratamentos, pois o processo de agrupamento é feito com base na distância de Mahalanobis. Para comparar as médias dos tratamentos, duas a duas, para cada variável, usaram-se os intervalos de confiança simultâneos de Bonferroni (Ferreira, 2003), que englobam os efeitos da combinação de todos tratamentos (dois capins e as cinco espécies arbóreas); assim, os efeitos dos capins no desempenho das variáveis foram também levados em consideração, inclusive por meio de sua combinação com as espécies arbóreas.

Os dados analisados, no presente experimento, referem-se às diferenças entre as variáveis avaliadas no início $(17 / 6 / 2005)$ e no final do experimento $(13 / 4 / 2007)$.

\section{Resultados e Discussão}

As duas primeiras variáveis canônicas explicaram, juntas, $76 \%$ da variação dos tratamentos (Tabela 1 ). Pela análise de variância dos dados obtidos pela primeira combinação linear (VC1) das seis variáveis, foram observadas diferenças significativas entre as médias dos dez tratamentos, pelo teste de Scott-Knott, a $5 \%$ de probabilidade. O resultado do teste indicou a formação de três agrupamentos.

Tabela 1. Escores das variáveis canônicas VC1 e VC2 e comparação entre as médias de $\mathrm{VC} 1$, dos tratamentos entre as combinações de leguminosas arbóreas introduzidas em pastagens estabelecidas de Brachiaria brizantha cv. Marandu e Panicum maximum cv. Tanzânia, na presença de gado.

\begin{tabular}{lcr}
\hline Tratamento & $\mathrm{VC}^{(1)}$ & \multicolumn{1}{c}{$\mathrm{VC} 2$} \\
\hline Mimosa artemisiana e capim-marandu & $16,1029 \mathrm{a}$ & 11,8692 \\
Mimosa artemisiana e capim-tanzânia & $16,9722 \mathrm{a}$ & 11,0801 \\
Pseudomanea guachapele e capim-marandu & $14,0186 \mathrm{c}$ & 14,5828 \\
Pseudomanea guachapele e capim-tanzânia & $15,0516 \mathrm{~b}$ & 14,4394 \\
Enterolobium contortisiliquum e capim-marandu & $15,7122 \mathrm{a}$ & 13,3402 \\
Enterolobium contortisiliquum e capim-tanzânia & $14,9389 \mathrm{~b}$ & 14,4987 \\
Acacia farnesiana e capim-marandu & $13,0736 \mathrm{c}$ & 9,2659 \\
Acacia farnesiana e capim-tanzânia & $16,5633 \mathrm{a}$ & 11,4800 \\
Mistura ${ }^{(2)}$ e capim-marandu & $16,3887 \mathrm{a}$ & 13,3599 \\
Mistura e capim-tanzânia & $16,1859 \mathrm{a}$ & 12,2522 \\
\hline Variância (\%) & 53 & 23 \\
Variância acumulada (\%) & 53 & 76 \\
\hline
\end{tabular}

${ }^{(1)}$ Médias seguidas por letras iguais não diferem entre si pelo teste de Scott-Knott, a $5 \%$ de probabilidade. ${ }^{(2)}$ Mistura das quatro espécies de leguminosas. 
Os tratamentos $M$. artemisiana e capins marandu e tanzânia, os de E. contortisiliquum e capim-marandu, de $A$. farnesiana e capim tanzânia, e a mistura de leguminosas e capins marandu e tanzânia apresentaram os maiores valores de VC1 e foram significativamente iguais; em seguida, destacaram-se os tratamentos $P$. guachapele e E. contortisiliquum, no pasto de capimtanzânia; e por último, significativamente iguais, os tratamentos $P$. guachapele e A. farnesiana em pasto de capim-marandu (Tabela 1).

Ao se comparar o desempenho das mudas sem proteção das três espécies arbóreas (M. artemisiana, $P$. guachapele e E. contortisiliquum), em pastagem de $B$. decumbens, na presença de gado, em Seropédica, RJ, Dias et al. (2007b) encontraram que M. artemisiana apresentou os maiores valores de $\mathrm{VC} 1$, nas avaliações dos períodos 24 a 28/9/2002 e 13 a 17/12/2002.

A espécie $M$. artemisiana apresentou a maior altura das mudas, após o pastejo $(86,2 \mathrm{~cm})$ no pasto do capim-marandu. Os tratamentos E. contortisiliquum e a mistura de leguminosas, no pasto de capim-marandu, apresentaram maior altura das mudas do que no pasto de capim-tanzânia, enquanto as mudas das espécies $P$. guachapele e A. farnesiana, apresentaram maior crescimento em altura no pasto de capim-tanzânia. Segundo Dias et al. (2007b), quando o objetivo é introduzir mudas das espécies arbóreas em pastagens existentes, o fato de elas não serem apreciadas pelo gado, fixarem $\mathrm{N}_{2}$ e apresentarem boa altura inicial representa vantagem na redução dos gastos com proteção para as mudas, no momento do plantio.

A correlação negativa $(r=-0,79 ; p=0,0035)$ entre altura da muda e pastejo indica que as mudas menos pastejadas apresentaram maior altura, enquanto a correlação positiva $(r=0,86 ; p=0,0007)$ entre altura da muda e diâmetro do caule e negativa $(r=-0,78$; $\mathrm{p}=0,0078)$ entre diâmetro do caule e pastejo confirmaram que a altura da muda e o diâmetro do caule decresceram com o pastejo (Tabela 2).

$\mathrm{Na}$ avaliação de aceitabilidade das plantas, é importante levar-se em consideração a preferência dos animais no pasto e se determinada planta já fez parte de sua dieta (Souto, 1967; Souto et al., 1975). Os animais usados no presente trabalho não tiveram, anteriormente, em sua dieta, as leguminosas usadas no experimento.

Segundo Launchbaugh et al. (1993), quando os animais encontram no pasto plantas que nunca entraram em sua dieta, inicialmente ingerem somente pequenas quantidades, e se nenhuma conseqüência negativa ocorrer, podem consumir essas plantas que, eventualmente, podem começar a fazer parte de sua dieta.

A baixa aceitabilidade de $M$. artemisiana, no presente trabalho, não ocorreu em razão da presença de acúleos na planta, uma vez que outras leguminosas testadas por Dias et al. (2007c) também apresentavam acúleos, como $M$. caesalpiniifolia, M. tenuiflora e Erythrina poeppigiana, que foram pastejadas pelos animais. Segundo Nozella (2001), muitas forrageiras usadas na alimentação de ruminantes possuem alto teor de proteína bruta $(16 \%)$, como a M. tenuiflora, mas apresentam baixa aceitabilidade, pois apresentam altos níveis de tanino (122 $\left.\mathrm{g} \mathrm{kg}^{-1}\right)$. $M$. artemisiana e $M$. tenuiflora quando comparadas com outras leguminosas arbóreas ( $P$. guachapele, E. contortisiliquum e outras 14 espécies), em pastagens de capim-marandu, B. decumbens e Cynodon sp. Tifton 85, apresentaram menor aceitabilidade pelos animais (Dias et al., 2007b).

Não foram observadas diferenças significativas, entre os dez tratamentos, quanto às variáveis diâmetro de caule e de copa, freqüência de pastejo e ocorrência de formiga, nas mudas das plantas (Tabela 3). Entretanto, maiores valores para diâmetro do caule $(1,76 \mathrm{~cm})$ e diâmetro da copa $(101,9 \mathrm{~cm})$ foram registrados no tratamento $M$. artemisiana e capim-marandu, e quanto à ocorrência de formiga $(21,3 \%)$ no tratamento E. contortisiliquum e capim-tanzânia. Estes resultados corroboram os de Ferreira et al. (2005), com relação às espécies arbóreas M. artemisiana e Samanea samanna ausência de gado -, e os de Dias et al. (2007c) com M. artemisiana, $P$. guachapele, E. contortisiliquum e

Tabela 2. Correlações de Pearson significativas entre as variáveis avaliadas de espécies de leguminosas arbóreas, introduzidas em pastagens estabelecidas de Brachiaria brizantha cv. Marandu e Panicum maximum cv. Tanzânia, na presença de gado.

\begin{tabular}{lrc}
\hline \multicolumn{1}{c}{ Variáveis } & \multicolumn{1}{c}{ r } & Significância de r \\
\hline Altura da muda (cm) vs. diâmetro do caule (cm) & 0,86 & 0,0007 \\
Altura da muda (cm) vs. pastejo (\%) & $-0,79$ & 0,0035 \\
Diâmetro do caule (cm) vs. sobrevivência (\%) & 0,69 & 0,0147 \\
Diâmetro do caule (cm) vs. pastejo (\%) & $-0,78$ & 0,0039 \\
Diâmetro do caule (cm) vs. formiga (\%) & $-0,59$ & 0,0351 \\
Diâmetro da copa (cm) vs. sobrevivência (\%) & 0,55 & 0,0481 \\
Diâmetro da copa (cm) vs. formiga (\%) & $-0,65$ & 0,0201 \\
Sobrevivência (\%) vs. formiga (\%) & $-0,69$ & 0,0144 \\
\hline
\end{tabular}


mais 13 espécies - na presença de gado -, quanto às variáveis diâmetro do caule e da copa, além dos de Dias et al. (2007a), quanto à ocorrência de formiga. Piagentini et al. (2002) avaliaram o estabelecimento de 18 espécies arbóreas, em áreas de rejeito, e destacaram que o aumento do diâmetro do caule de algumas árvores foi decisivo para que elas se desenvolvessem em ambientes adversos.

As correlações negativas de diâmetro do caule e diâmetro da copa, com ocorrência de formigas $(r=-0,59$; $\mathrm{p}=0,0351$ e $\mathrm{r}=-0,65 ; \mathrm{p}=0,0201$, respectivamente), mostraram que quanto menor os diâmetros do caule e da copa das mudas das quatro leguminosas, menores foram as ocorrências de formigas nas plantas (Tabela 2).

Em relação à sobrevivência das mudas, as maiores percentagens foram as da espécie $M$. artemisiana e da mistura de arbóreas, em ambos os pastos; E. contortisiliquum em capim-marandu e A. farnesiana em capim-tanzânia. No tratamento $P$. guachapele e capim-tanzânia, foi observada a maior percentagem de sobrevivência de mudas (Tabela 3). As mudas com maiores diâmetros de caule e copa apresentaram maior sobrevivência, enquanto as mudas com maior ocorrência de formigas tiveram as menores percentagens de sobrevivência (Tabela 2).

De modo geral, a espécie $M$. artemisiana, em pasto de capim-marandu, foi a leguminosa que melhor se estabeleceu na arborização da pastagem, sem a retirada do gado e sem a proteção das mudas. Isso porque as mudas dessa espécie, no pasto de capim-marandu, apresentaram melhor crescimento (maior altura da muda) e maior percentagem de sobrevivência. Este tratamento, apesar de não ter sido significativo, apresentou maiores valores de diâmetro de caule
$(1,76 \mathrm{~cm})$, diâmetro de copa $(101,9 \mathrm{~cm})$, e menores valores quanto à freqüência de pastejo $(13,3 \%)$ e ocorrência de formigas, o que consolida sua indicação para a região como a espécie de leguminosa a ser introduzida com sucesso, em pastagem de capim-marandu, em presença de gado.

O melhor desempenho de M. artemisiana, no pasto de capim-marandu, pode ser atribuído ao maior desenvolvimento do capim-tanzânia, que abafa mais as mudas dessa leguminosa, ou à maior aceitabilidade do capim-marandu pelos animais. Essas possibilidades foram observadas durante o período experimental; assim, esse melhor desempenho deve ser atribuído à ação simultânea desses efeitos.

Outras qualidades são atribuídas à M. artemisiana, como por exemplo: reabilitar solos degradados (Dias et al., 1995) e recuperar fertilidade de solos (Foletti et al., 1992), favorecer a ocorrência do grupo Oligochaeta no solo (Dias et al., 2007a), crescer e nodular em ambiente com sombreamento denso (Ferreira et al., 2005), favorecer a fixação de $\mathrm{N}$ atmosférico de capins sob sua copa (Dias, 2005), gerar madeira com melhor aproveitamento para a geração de energia, em comparação ao Eucalyptus grandis (Paula, 1995), e produzir quantidade considerável de serapilheira (Dias et al., 2007b).

As mudas de $P$. guachapele e A. farnesiana apresentaram maior altura e sobrevivência no pasto do capim-tanzânia, enquanto os maiores valores em relação a essas variáveis foram encontrados no pasto do capim-marandu, nos tratamentos E. contortisiliquum e mistura. Portanto, para a introdução dessas espécies nos pastos, sem a proteção das mudas, é importante seguir as recomendações sugeridas por Carvalho et al.

Tabela 3. Variáveis avaliadas dos tratamentos entre as combinações de leguminosas arbóreas introduzidas em pastagens estabelecidas de Brachiaria brizantha cv. Marandu e Panicum maximum cv. Tanzânia, na presença de gado ${ }^{(1)}$.

\begin{tabular}{|c|c|c|c|c|c|c|}
\hline Tratamento & $\begin{array}{l}\text { Altura da } \\
\text { muda }(\mathrm{cm})\end{array}$ & $\begin{array}{c}\text { Diâmetro do } \\
\text { caule }(\mathrm{cm})\end{array}$ & $\begin{array}{c}\text { Diâmetro da } \\
\text { copa }(\mathrm{cm})\end{array}$ & $\begin{array}{c}\text { Sobrevivência } \\
\text { da muda }(\%)\end{array}$ & $\begin{array}{c}\text { Freqüência de } \\
\text { pastejo }(\%)\end{array}$ & $\begin{array}{c}\text { Ocorrência de } \\
\text { formiga }(\%)\end{array}$ \\
\hline Mimosa artemisiana e capim-marandu & $86,2 \mathrm{a}$ & 1,76 & 101,9 & $98,7 \mathrm{a}$ & 13,3 & 4,0 \\
\hline Mimosa artemisiana e capim-tanzânia & $19,2 \mathrm{~g}$ & 0,86 & 74,1 & $97,3 \mathrm{ab}$ & 41,3 & 5,3 \\
\hline Pseudomanea guachapele e capim-marandu & $46,9 \mathrm{~d}$ & 0,68 & 4,9 & $80,0 \mathrm{c}$ & 32,0 & 17,3 \\
\hline Pseudomanea guachapele e capim-tanzânia & $51,3 \mathrm{c}$ & 0,82 & 6,8 & $89,3 b$ & 29,3 & 12,0 \\
\hline Enterolobium contortisiliquum e capim-marandu & $66,2 b$ & 1,68 & 16,7 & $94,7 \mathrm{ab}$ & 16,0 & 5,3 \\
\hline Enterolobium contortisiliquum e capim-tanzânia & $12,9 \mathrm{~h}$ & 0,30 & 6,4 & $78,7 \mathrm{c}$ & 37,3 & 21,3 \\
\hline Acacia farnesiana e capim-marandu & $30,8 \mathrm{f}$ & 0,73 & 54,5 & $78,7 \mathrm{c}$ & 29,3 & 2,7 \\
\hline Acacia farnesiana e capim-tanzânia & $36,3 \mathrm{e}$ & 1,01 & 79,9 & $96,0 \mathrm{ab}$ & 18,7 & 5,3 \\
\hline Mistura $^{(2)}$ e capim-marandu & $45,0 \mathrm{~d}$ & 1,04 & 40,3 & $94,0 \mathrm{ab}$ & 36,0 & 6,0 \\
\hline Mistura e capim-tanzânia & $36,6 \mathrm{e}$ & 0,98 & 24,6 & $100,0 \mathrm{a}$ & 32,0 & 0,0 \\
\hline
\end{tabular}

${ }^{(1)}$ Médias (de três repetições) seguidas por letras iguais não diferem entre si pelo teste de Scott-Knott, a 5\% de probabilidade. ${ }^{(2)}$ Mistura das quatro espécies de leguminosas. 
(1998), como o plantio de mudas dessas espécies associado a culturas anuais, retardando-se a semeadura das forrageiras por um ou dois anos, o que reduz a necessidade de proteção das mudas. Baggio \& Carpanezzi (1989) relataram que os melhores resultados foram obtidos quando foram usadas mudas altas, aliada aos métodos de proteção. Isso em razão de algumas espécies arbóreas apresentarem alta palatabilidade, valor forrageiro e servirem, principalmente, para suplementação da alimentação dos animais, em período de escassez de forragem.

\section{Conclusões}

1. Mimosa artemisiana é indicada para ser introduzida com nas pastagens de Brachiaria brizantha cv. Marandu, sem a proteção das mudas com cercas ou estacas, e em presença do gado.

2. Pseudomanea guachapele e Acacia farnesiana são indicadas para ser introduzidas nas pastagens de capim-tanzânia, com proteção das mudas e na presença de gado.

3. Enterolobium contortisiliquum e a mistura das quatro espécies de leguminosas são indicadas para ser introduzidas nas pastagens de capim-marandu, com proteção das mudas e na presença de gado.

\section{Referências}

ALONZO, Y.M. Potential of silvopastoral systems for economic dairy production in Cayo, Belize and constraints for their adoption. 2000. 81p. Tesis (M.Sc.) - Universidad de Costa Rica, Turrialba.

ANDERSSON, M.S.; SCHULTZE-KRAFT, R.; PETERS, M.; HINCAPIÉ, B.; LASCANO, C.E. Morphological, agronomic and forage quality diversity of the Flemingia macrophylla world collection. Field Crops Research, v.96, p.387-406, 2006.

ANDRADE, C.M.S.; VALENTIM, J.F.; CARNEIRO, J.C. Árvores de baginha (Stryphnodendron guianense) em ecossistemas de pastagens cultivadas na Amazônia Ocidental. Revista Brasileira de Zootecnia, v.31, p.1-5, 2002.

ASH, A.J. The effect of supplementation with leaves from the leguminous trees Sesbania grandiflora, Albizia chinensis and Gliricidia sepium on the intake digestibility of guinea grass hay by goats. Animal Feed Science and Technology, v.28, p.225-232, 1990.

BAGGIO, A.J.; CARPANEZZI, O.B. Resultados preliminares de um estudo sobre arborização de pastagens com mudas de espera. Curitiba: Embrapa Floresta, 1989. 22p. (Embrapa Floresta. Boletim de Pesquisa Florestal, 19).
BAUMONT, R.; PRACHE, S.; MEURET, M.; MORAND-FEHR, P. How forage characteristics influence behavior and intake in small ruminants: a review. Livestock Production Science, v.64, p. $15-28,2000$.

CARVALHO, M.M. Arborização de pastagens cultivadas. Juiz de Fora: Embrapa-CNPGL, 1998. 37p. (Embrapa-CNPGL. Documentos, 64).

DE-POLLI, H.; FRANCO, A.A. Inoculação de sementes de leguminosas. 1985. 31p. (Embrapa-UAPNBS. Circular Técnica, 1).

DIAS, L.E.; FRANCO, A.A.; CAMPELLO, E.F.; FARIA, S.M.; SILVA, E.M. Forest legumes: aspects related to their nutrition and use in reclamation of degraded soils. Bosque, v.16, p.121-127, 1995.

DIAS, P.F. Importância da arborização de pastagens com leguminosas fixadoras de nitrogênio. 2005.128p. Tese(Doutorado) - Universidade Federal Rural do Rio de Janeiro, Seropédica.

DIAS, P.F.; SOUTO, S.M.; CORREIA, M.E.F.; ROCHA, G.P.; MOREIRA, J.F.; RODRIGUES, K. de M.; FRANCO, A.A. Árvores fixadoras de nitrogênio e macrofauna do solo em pastagem de híbrido de Digitaria. Pesquisa Agropecuária Brasileira, v.41, p.1015-1021, 2006.

DIAS, P.F.; SOUTO, S.M.; CORREIA, M.E.F.; RODRIGUES, K. de M.; FRANCO, A.A. Efeito de leguminosas arbóreas sobre a macrofauna do solo em pastagem de Brachiaria brizantha cv. Marandu. Pesquisa Agropecuária Tropical, v.37, p.38-44 $2007 \mathrm{a}$.

DIAS, P.F.; SOUTO, S.M.; COSTA, J.R. Análise do comportamento de espécies leguminosas arbóreas introduzidas em pastagens de gramíneas tropicais. Pesquisa Agropecuária Tropical, v.37, p.31-37, 2007b.

DIAS, P.F.; SOUTO, S.M.; FRANCO, A.A. Leguminosas arbóreas introduzidas em pastagem. Pesquisa Agropecuária Brasileira, v.42, p.119-126, 2007c.

DIAS FILHO, M.B.; FERREIRA, J.N. Barreiras para adoção de sistemas silvipastoris. In: SIMPÓSIO DE FORRAGICULTURA E PASTAGEM, 6., 2007, Lavras. Forragicultura e pastagem: temas em evidência: relação custo benefício. Lavras: Ufla, 2007, p.347-365.

FARIA, S.M. de. Obtenção de estirpes de rizóbio eficientes na fixação de nitrogênio para espécies florestais (aproximação 2001). Seropédica: Embrapa Agrobiologia, 2001. 21p. (Embrapa Agrobiologia. Documentos, 134).

FERREIRA, D.E. Análise de variância multivariada. In: FERREIRA, D.E. (Ed.). Estatística multivariada. Lavras: Ufla, 2003. p.218-231. Disponível em: $<$ http://www.dex.ufla. br/ danielff/dex522.pdf $>$. Acesso em: 31 ago. 2003.

FERREIRA, D. de J.; DIAS, P.F.; SOUTO, S.M.; JIMENEZ, L. Efeito do sombreamento no estabelecimento de leguminosas arbóreas para pastagens. Pasturas Tropicales, v.27, p.38-44, 2005.

FOLETTI, C.; KASS, D.; LANDAVERDE, R.; NOLASCO, R.; FELKER, R.; SZOTT, L. Improved fallows in traditional agroforestry systems in Central America. Agroforestería, v.7, p.8, 1992. 
FUNDAÇÃO ARTHUR BERNARDES. Sistema para análises estatísticas: SAEG 9.0. Viçosa, 2005. 1 CD-ROM.

HINDRICHSEN, I.K.; OSUJI, P.O.; ODENYO, A.A.; MADSEN, J.; HVELPLUND, T. Effect of supplementation of maize stover with foliage of various tropical multipurpose trees and Lablab purpureus on intake, rumen fermentation, digesta kinetics and microbial protein supply of sheep. Animal Feed Science and Technology, v.113, p.83-96, 2004.

IBRAHIM, M.; SCHLONVOIGGT, A.; CAMARGO, C.; SOUZA, M. Multistrata silvopastoral systems for incresing productivity and conservation of natural resources in Central America. In: INTERNATIONAL GRASSLAND CONGRESS, 19., 2001, Brasília. Proceedings. Brasília: Embrapa, 2001. p.645-649.

LAUNCHBAUGH, K.L.; PROVENZA, F.D.; BURRITT, E.A. How herbivores track variable environments: response to variability of phytotoxins. Journal of Chemical Ecology, v.19, p.1047-1056, 1993.

MACEDO, M.O; RESENDE, A.S.; GARCIA, P.C.; BODDEY, R.M.; JANTALIA, C.P.; URQUIAGA, S.; CAMPELLO, E.F.C.; FRANCO, A.A. Changes in soil $\mathrm{C}$ and $\mathrm{N}$ stocks and nutrient dynamics 13 years after recovery of degraded land using leguminous nitrogen-fixing trees. Forest Ecology and Management, v.255, p.1516-1524, 2008.

MONTOYA,L.J.;BAGGIO,A.J.Estudos econômicos da introdução de mudas altas para sombreamento de pastagens. In: ENCONTRO BRASILEIRO DE ECONOMIA E PLANEJAMENTO FLORESTAL, 1., 1991, Curitiba. Anais. Curitiba: Embrapa Floresta, 1991. v.2, p.172-191.

NICODEMO, M.L.F.; SILVA, V.P. da; THIAGO, L.R.L. de S.; GONTIJO NETO, M.M.; LAURA, V.A. Sistemas silvipastoris: introdução de árvores na pecuária do Centro-Oeste brasileiro. Campo Grande: Embrapa Gado de Corte, 2004. 37p. (Embrapa Gado de Corte. Documentos, 146).
NOZELLA, E.F. Determinação de taninos em plantas com potencial forrageiro para ruminantes. 2001. 58p. Dissertação (Mestrado) - Universidade de São Paulo, Piracicaba.

NOZELLA, E.F.; CABRAL FILHO, S.L.S.; BUENO, I.C. da S.; GODOY, P.B.; LONGO, C.; BORGES, J.H.; VITTI, D.M.S.S. Caracterização de forrageiras do Nordeste utilizando a técnica de produção de gases, composição química e quantificação de taninos. 1. Plantas do Estado da Bahia. In: REUNIÃO ANUAL DA SOCIEDADE BRASILEIRA DE ZOOTECNIA, 43., 2006, João Pessoa. Anais. João Pessoa: SBZ, 2006. CD-ROM.

PAULA, J.E. de. Anatomia e dendrometria de Mimosa artemisiana e Eucalyptus grandis. Pesquisa Agropecuária Brasileira, v.30, p.745-757, 1995.

PIAGENTINI, P.M.; DIAS, L.E.; CAMPELLO, E.F.C.; RIBEIRO JUNIOR, E.S. Crescimento de diferentes espécies arbóreas e arbustivas em depósito de rejeito de beneficiamento de minérios de zinco em vazante. In: SIMPÓSIO NACIONAL SOBRE RECUPERAÇÃO DE ÁREAS DEGRADADAS, 5., 2002, Belo Horizonte. Anais. Belo Horizonte: Sobrade, 2002. p.413-419.

PIMENTEL-GOMES, F. Curso de estatística experimental. 14.ed. Piracicaba: Nobel, 2000. 430p.

RIBEIRO JUNIOR, J.I. Análises estatísticas no SAEG. Viçosa: UFV, 2001. 301p.

SOUTO, S.M. Aceitabilidade e persistência de forrageiras tropicais. In: REUNIÃO ANUAL DA SOCIEDADE BRASILEIRA DE ZOOTECNIA, 7., 1967, Piracicaba. Anais. Piracicaba: SBZ, 1967. p.11-14.

SOUTO, S.M.; LIMA, C.R.; LUCAS, E.D. Palatabilidade de leguminosas forrageiras. Pesquisa Agropecuária Brasileira, v.10, p.7-11, 1975.

Recebido em 27 de março de 2008 e aprovado em 16 de setembro de 2008 